\title{
Measurement of the Nonlinear Coefficient of a Second-Higher Term in Lead Zirconate Titanate Piezoelectric Ceramics with Half-Surface Electrodes
}

\author{
Shinjiro TASHIRO, Wataru TOKUNAGA, Keisuke ISHII and Kunihiro NAGATA \\ Department of Materials Science and Engineering, The National Defense Academy, 1-10-20, Hashirimizu, Yokosuka-shi 239-8686 \\ 半面電極試料を用いた PZT 圧電セラミックスの 2 次項非線形定数測定 \\ 田代新二郎·徳永 亘·石井啓介·永田邦裕 \\ 防衛大学校機能材料工学科, 239-8686 神奈川県横須賀市走水 1-10-20
}

\begin{abstract}
Second-harmonic voltage between a pair of electrodes, appearing due to nonlinearity, was theoretically calculated in piezoelectric rectangular vibrators with a pair of half electrodes which were driven by a sinusoidal constant current having the resonance frequency of length-extensional $1 / 2 \lambda$-mode vibration. The theoretical calculation was performed using the electrical equivalent circuit of a piezoelectric rectangular vibrator. A material constant as a nonlinear coefficient of second-higher term can be derived by using an equation, as that obtained in the present paper, showing the relationship between the magnitude of second harmonic voltage and the nonlinear coefficient of second-higher term. [Received October 26, 2004; Accepted January 20, 2005]
\end{abstract}

Key-words : Nonlinear piezoelectric coefficient, Lead zirconate titanate, High-power vibration, Piezoelectric vibrator, Half electrodes surface

\section{Introduction}

Nonlinear phenomena have become serious problems that prevent operation stability and high efficiency in piezoelectric ceramics used in electronic devices for high-power applications, because of the downscaling of electronic devices and increase of their power density. The current jump phenomenon and harmonic voltage generations are typical nonlinear phenomena that are observed in piezoelectric vibrators driven with a high power at around the resonance frequency. ${ }^{1-4)}$

In order to quantitatively analyze the nonlinearity in piezoelectric ceramics, it is necessary to obtain nonlinear coefficients. Although several researchers have published papers on nonlinear coefficients of lead zirconate titanate (PZT), since the frequencies were less than $100 \mathrm{~Hz}$ in those studies, ${ }^{5)-8)}$ it is difficult to apply those results directly to piezoelectric ceramics driven at the resonance frequency.

On the other hand, several researchers have analyzed the nonlinear phenomena in high-power vibrations in terms of the change of linear constants, from the viewpoint of practical use. ${ }^{9)-12)}$ However, since these analyses focused on the change of linear constants, it is necessarily impossible to define the nonlinear material constants that quantitatively represent nonlinearity.

In contrast to the studies on nonlinearity mentioned above, the principal objectives of our study are to define nonlinear material constants that quantitatively represent nonlinearity and to introduce the equation of the relationship between voltage and current representing quantitatively nonlinear phenomena arising with high-power vibrations. We already reported the theoretical analysis about the relationship between the nonlinear coefficient of a third-higher term, $\xi_{\mathrm{D} 31}$, and the third-harmonic voltage generating between electrodes when a rectangular ceramic with whole electrodes on both upper and lower surfaces was driven by a sinusoidal constant current having the resonance frequency of length-extensional $1 / 2 \lambda$-mode vibration. ${ }^{13)}$

The $\xi_{\mathrm{D} 31}$ value rules over the current jump phenomenon which is one of the typical nonlinear phenomena. It is there- fore possible to theoretically calculate the driving conditions causing the current jump phenomenon, if a $\xi_{\mathrm{D} 31}$ value is obtained. ${ }^{14)}$ Because of these circumstances, we have investigated about the nonlinear coefficient of a third-higher term. On the other hand, the nonlinear coefficient of a secondhigher term rules over the generation of the second harmonic voltage which is one of the nonlinear voltages. Since the second harmonic voltage has twice frequency of driving frequency, when a rectangular bar vibrator with half electrodes was driven in high power levels at resonance frequency, the frequency of the second harmonic voltage generating in the sample belong consequently in the frequency range of the second harmonic resonance. It is therefore anticipated that the influence of the second harmonic voltage becomes remarkable in the sample with half electrodes. Because of these circumstances, it is necessary to establish the measurement method also about the nonlinear-coefficient of second higher term. In the present study, we analyze the relationship between the second harmonic voltage and the nonlinear coefficient of second-higher term, using the electrical equivalent circuit, in a piezoelectric rectangular bar with a pair of half electrodes on both upper and lower surfaces. The nonlinear coefficient of second-higher term can be successively derived as a material constant in this analysis.

\section{Nonlinear piezoelectric equation}

As the nonlinear piezoelectric equations of a rectangular vibrator with whole surface electrodes as shown in Fig. 1 (a), Eqs. (1) and (2) were proposed in the previous paper. ${ }^{13)}$

$$
\begin{gathered}
E_{3}=-\frac{g_{31}}{S_{11}{ }^{D}} S_{1}+\beta_{33}{ }^{S} D_{3}+\gamma_{\mathrm{D} 31} D_{3}{ }^{2}+\xi_{\mathrm{D} 31} D_{3}{ }^{3} \\
T_{1}=\frac{1}{S_{11}{ }^{D}} S_{1}-\frac{g_{31}}{s_{11}{ }^{D}} D_{3}+\chi_{\mathrm{D} 31} D_{3}{ }^{2}+\zeta_{\mathrm{D} 31} D_{3}{ }^{3}
\end{gathered}
$$

Since Eqs. (1) and (2) are nonlinear piezoelectric equations, it is difficult to obtain the relationship between voltage and current by solving exactly them. Equations (1) and (2) were 


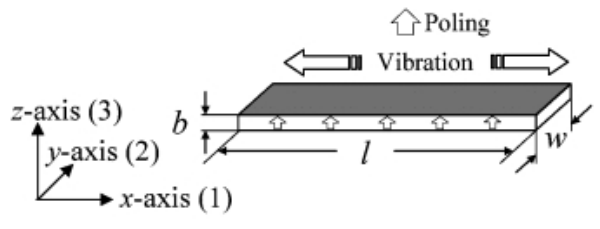

(a)

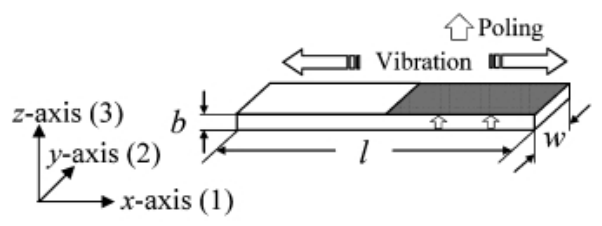

(b)

Fig. 1. Configuration of the rectangular bar vibrators $(l=29.5 \mathrm{~mm}$, $w=2.62-5.93 \mathrm{~mm}, b=0.9 \mathrm{~mm}$ ) with whole surface electrodes (a) and half electrodes (b).

therefore solved using an approximation as follows. The relationship between linear terms is independent even when nonlinear phenomena occur. Nonlinear voltages occurring in the sample can simply be added to the voltage of linear terms. This is the superposition between linear and nonlinear terms, as well as the perturbation of nonlinear terms to linear ones. That is, this is an approximation by perturbation, which is applied when nonlinear terms are small compared with linear terms.

Because of driving at around the resonance frequency, since the current flowing through the damping capacitance, $C_{s}$, is very small compared with that flowing through the motional impedance, the current flowing through $C_{\mathrm{s}}$ can be neglected. By adding nonlinear terms to the solution of the linear terms according to the approximation by perturbation mentioned above, we obtain

$$
\begin{aligned}
v_{3}= & L_{1} \frac{\mathrm{d} i_{3}}{\mathrm{~d} t}+R_{1} i_{3}+\frac{1}{C_{1}} \int i_{3} \mathrm{~d} t+\gamma_{\mathrm{D} 31}^{\prime}\left(\int i_{3} \mathrm{~d} t\right)^{2} \\
& +\xi_{\mathrm{D} 31}^{\prime}\left(\int i_{3} \mathrm{~d} t\right)^{3}
\end{aligned}
$$

where $v_{3}$ is the voltage between electrodes, $i_{3}$ is the current, $L_{1}$, $C_{1}$ and $R_{1}$ are equivalent-circuit constants, and $\gamma_{\mathrm{D} 31}^{\prime}$ and $\xi_{\mathrm{D} 31}^{\prime}$ are effective nonlinear coefficients for the vibrator. The effective nonlinear coefficients are constants for each vibrator, and change with the size of the vibrator.

When the sample is driven with a constant current of $i_{3}=I_{3}$ $\sin \omega t$ at the resonance frequency, the voltage between electrodes, $v_{3}$, is obtained by substituting $i_{3}=I_{3} \sin \omega t$ into Eq. (3).

$$
\begin{aligned}
v_{3}= & \left(\omega L_{1}-\frac{1}{\omega C_{1}}\right) I_{3} \cos \omega t+R_{1} I_{3} \sin \omega t \\
& +\frac{\gamma_{\mathrm{D} 31}^{\prime}}{\omega^{2}} I_{3}{ }^{2} \cos ^{2} \omega t-\frac{\xi_{\mathrm{D} 31}^{\prime}}{\omega^{3}} I_{3}{ }^{3} \cos ^{3} \omega t
\end{aligned}
$$

Expanding $\cos ^{2} \omega t$ and $\cos ^{3} \omega t$ using trigonometric formulae for double and triple angles, Eq. (4) is rewritten as

$$
v_{3}=\frac{\gamma_{\mathrm{D} 31}^{\prime}}{2 \omega^{2}} I_{3}^{2}
$$

$$
\begin{aligned}
& +\left(\omega L_{1}-\frac{1}{\omega C_{1}}\right) I_{3} \cos \omega t+R_{1} I_{3} \sin \omega t \\
& -\frac{3 \xi_{\mathrm{D} 31}^{\prime}}{4 \omega^{3}} I_{3}{ }^{3} \cos \omega t+\frac{\gamma_{\mathrm{D} 31}^{\prime}}{2 \omega^{2}} I_{3}{ }^{2} \cos 2 \omega t \\
& -\frac{\xi_{\mathrm{D} 31}^{\prime}}{4 \omega^{3}} I_{3}{ }^{3} \cos 3 \omega t .
\end{aligned}
$$

A dc component and $2 \omega$ component due to $\gamma_{\mathrm{D} 31}^{\prime}$, and a $\omega$ component and $3 \omega$ component due to $\xi_{\mathrm{D} 31}^{\prime}$, are generated as nonlinear voltages, respectively. We have quantitatively expressed nonlinear phenomena using Eq. (5). ${ }^{1)-4)}$

\section{Experimental procedures}

In the measurement of the nonlinear coefficient of thirdhigher term, $\xi_{\mathrm{D} 31}$, a sample with whole electrodes was used in Fig. 1 (a). When the sample is driven with a constant current at the fundamental resonance angular frequency $\left(\omega_{r}=2 \pi f_{\mathrm{r}}\right)$, the angular frequency of the third-harmonic voltage appearing between electrodes due to $\xi_{\mathrm{D} 31}$ is $3 \omega_{r}$. Since the sample with whole electrodes has many resonance frequencies at odd number times of fundamental resonance frequency, the third-harmonic resonance occurs at around $3 \omega_{r}$. The magnitude of third-harmonic voltage appearing between electrodes was significantly influenced by the position of $3 \omega_{r}$ on the third-harmonic resonance curve. The magnitude of third-harmonic voltage appearing between electrodes showed the maximum when $3 \omega_{r}$ coincided exactly with the anti-resonance angular frequency $\left(\omega_{3 \mathrm{a}}=2 \pi f_{3 \mathrm{a}}\right)$ of the third-harmonic resonance. Thus, the motional impedance at $3 \omega_{r}$ was essential to calculate $\xi_{\mathrm{D} 31}$ value from the magnitude of third-harmonic voltage. ${ }^{13)}$

Analogizing from the theoretical analysis used in derivation of $\xi_{\mathrm{D} 31}$, it is necessary that a piezoelectric resonance exists at around $2 \omega_{r}$ to derive the nonlinear coefficient of secondhigher term, $\gamma_{\mathrm{D} 31}$, from the magnitude of second-harmonic voltage. Since a sample with half electrodes shown in Fig. 1 (b) has a piezoelectric resonance at around $2 \omega_{r}$, the sample with half electrodes was used in the estimation of $\gamma_{\mathrm{D} 31}$.

The composition of the prepared samples is $0.95 \mathrm{~Pb}$ $\left(\mathrm{Zr}_{0.51} \mathrm{Ti}_{0.49}\right) \mathrm{O}_{3}-0.05 \mathrm{~Pb}\left(\mathrm{Sb}_{0.5} \mathrm{Nb}_{0.5}\right) \mathrm{O}_{3}-0.015 \mathrm{MnO}$. The samples were prepared using oxide powders as starting materials, by means of a conventional method, namely, mixing by wet ball-milling, calcining at $850^{\circ} \mathrm{C}$ for $2 \mathrm{~h}$ in air, grinding by wet ball-milling, pressing at $100 \mathrm{MPa}$, and firing at $1240^{\circ} \mathrm{C}$ for $2 \mathrm{~h}$ in air. After firing silver electrodes onto the upper and lower half surfaces of the rectangular bars as shown in Fig. 1(b), the samples were poled under an electric field of $3 \mathrm{kV} / \mathrm{mm}$ for $10 \mathrm{~min}$ in a silicon oil bath kept at $120^{\circ} \mathrm{C}$. Mechanical quality factor, $Q_{\mathrm{m}}$, electromechanical coupling factor, $k_{31}$, piezoelectric $d_{31}$ and $h_{31}$ constants, elastic compliance, $s_{11}{ }^{E}$, and relative permittivity, $\varepsilon_{33}{ }^{T} / \varepsilon_{0}$, are listed in Table 1 . These material constants were derived from the resonance-antiresonance characteristic of a whole electrodes sample in Fig. 1(a) measured at a low current level of less than $1 \mathrm{~mA}$ using an LF impedance analyzer (Hewlett-Packard, 4192A).

The second-harmonic voltage was measured using the constant-current circuit shown in Fig. 2. In constant-current driving, the sample should be driven by a sinusoidal current without any distortion. A power amplifier (NF Electronics Instruments, 4020) generates a second-harmonic voltage with a magnitude of 0.1 to $0.4 \%$ of the fundamental voltage; driving current consequently imposes a distortion via the secondharmonic voltage. In order to prevent the application of the second-harmonic voltage to the sample, a parallel resonance 
Table 1. Piezoelectric Constants of the Sample

\begin{tabular}{|c|c|}
\hline$Q_{m}$ & 1550 \\
\hline$k_{31}$ & 0.366 \\
\hline$d_{31}\left(\times 10^{-12} \mathrm{~m} / \mathrm{V}\right)$ & 121 \\
\hline$h_{31} \quad\left(\times 10^{9} \quad \mathrm{~N} / \mathrm{C}\right)$ & 1.27 \\
\hline$S 11^{\mathrm{E}} \quad\left(\times 10^{-12} \mathrm{~Pa}^{\cdot 1}\right)$ & 11.4 \\
\hline$\varepsilon 33^{\mathrm{T} / \varepsilon 0}$ & 1270 \\
\hline
\end{tabular}

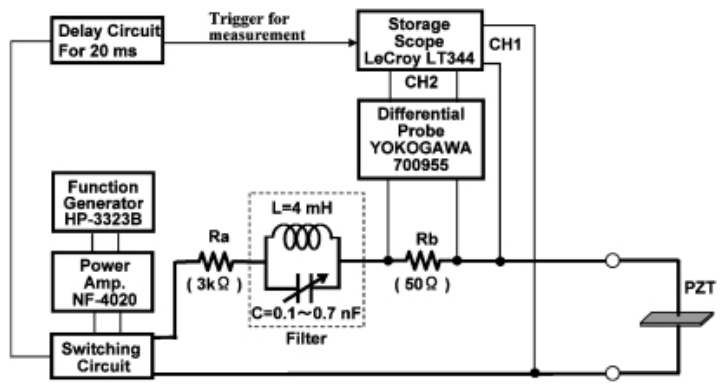

Fig. 2. Measuring circuit used for the constant-current driving.

circuit was inserted in series with the sample, as shown in Fig. 2. The resonance frequency of the parallel resonance circuit was adjusted to a frequency equal to two times the driving frequency. Since the parallel resonance circuit has an impedance above $100 \mathrm{k} \Omega$ at the frequency of the second-harmonic voltage of the power amplifier (equal to the frequency of the second-harmonic voltage generated in the sample), the parallel resonance circuit prevents the application of the second-harmonic voltage generated in the power amplifier to the sample, and simultaneously enables us to take the secondharmonic voltage, generating in the sample, out of the sample.

The series resistors, $R_{\mathrm{a}}$ and $R_{\mathrm{b}}$, were used for the realization of constant-current driving and for monitoring the current. The current was kept constant by the series resistor $R_{\mathrm{a}}$ of $3 \mathrm{k} \Omega$ during the measurement, because the resonance resistance of the samples was less than $20 \Omega$ at the resonance frequency. A resistor of $50 \Omega$ was used as $R_{\mathrm{b}}$. The frequency of a function generator (Hewlett-Packard, 3323B) was fixed at a fundamental resonance frequency of the $1 / 2 \lambda$-mode of the sample in Fig. 1(b), and the signal of the function generator was amplified by a power amplifier (NF Electronics Instruments, 4020). A spectrum analyzer (Hewlett-Packard, ESAL1500A) was used for the measurement of the magnitudes of fundamental and second-harmonic voltages between electrodes of the sample.

\section{Results and discussion}

The $f_{2 \mathrm{a}}$ is an anti-resonance frequency in the second-harmonic resonance. The second-harmonic resonance curve in Fig. 3 (a) is representative of the curves of six samples differing slightly in the relation between $f_{\mathrm{r}}$ and $f_{2 \mathrm{a}}$, namely, showing the curve on the basis of $f_{2 a}$, it is showed as Fig. $3(\mathrm{a})$. Since six resonance curves almost override one another, it is showed for convenience with a curve being representative. The slight differences in the relation between $f_{\mathrm{r}}$ and $f_{2 \mathrm{a}}$ were caused by the slight change of the size and configuration of samples. When the six samples with the configuration shown in Fig. 1 (b) are driven at the fundamental resonance frequency by the constant-current method, the impedance at two times the driving frequency shows various values on the second-harmonic

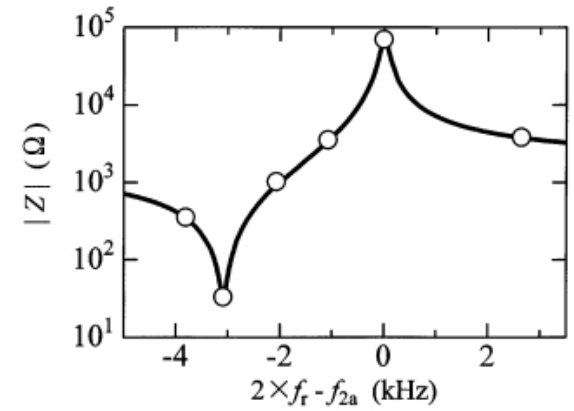

(a)

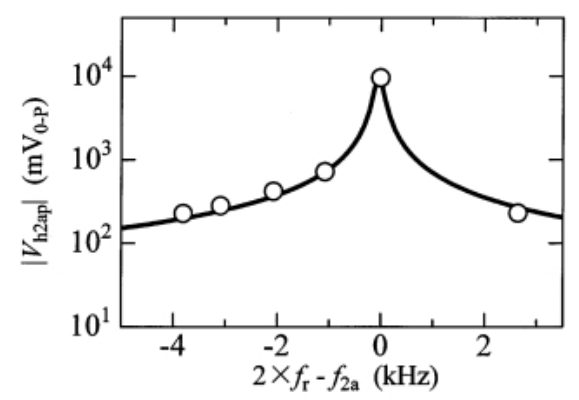

(b)

Fig. 3. Impedance values of six samples at $2 \times f_{\mathrm{r}}$ on the second-harmonic resonance curve which is representative of the curves of six samples for convenience (a), and the magnitudes of second-harmonic voltage between electrodes of the six samples (b). The second-harmonic resonance and second-harmonic antiresonance frequencies, $f_{2 \mathrm{r}}$ and $f_{2 \mathrm{a}}$, are about $117 \mathrm{kHz}$ and $120 \mathrm{kHz}$, respectively.

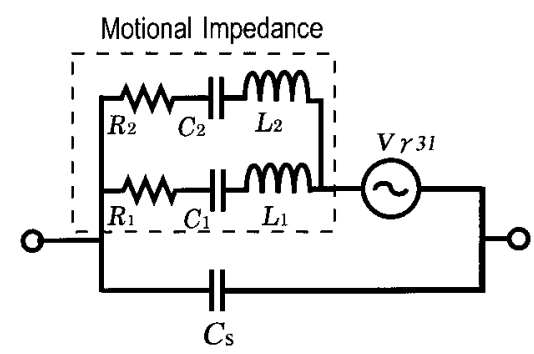

Fig. 4. Equivalent circuit of a piezoelectric vibrator with half electrodes. The second-harmonic nonlinear voltage source is included.

resonance curve, as shown in Fig. 3 (a). The plotted circles in Fig. 3 (a) show where $2 \times f_{\mathrm{r}}$ occupies on the second-harmonic resonance curve.

Figure 3(b) shows the magnitude of second-harmonic voltages, $V_{\mathrm{h} 2 a p}$, between a pair of electrodes when these six samples were driven at the fundamental resonance frequency by the constant current of $10 \mathrm{~mA}_{0-\mathrm{P}}$. When $2 \times f_{\mathrm{r}}$ agreed with $f_{2 \mathrm{a}}$, the maximum $V_{\text {h2ap }}$ was measured. This fact is the same circumstances as the third-harmonic voltage generating in the whole electrodes sample in Fig. 1(a). ${ }^{13)}$ The electrical equivalent circuit including nonlinear term of a piezoelectric ceramic can be therefore composed of motional impedance parameters originating in fundamental and second-harmonic resonances ( $L_{1}, C_{1}, R_{1}, L_{2}, C_{2}, R_{2}$ ), damping capacitance, $C_{\mathrm{s}}$, and the nonlinear voltage source $v_{\gamma 31}$. Figure 4 is the equivalent circuit including $v_{\gamma 31}$.

The magnitude of second-harmonic voltage appearing between electrodes, $V_{\text {h2ap }}$, can be expressed as Eq. (6) by con- 
sidering the equivalent circuit and the magnitude of the $2 \omega$ component in Eq. (5).

$$
V_{\mathrm{h} 2 \mathrm{ap}}=\frac{\gamma_{\mathrm{D} 31}^{\prime} I_{3}^{2}}{2 \omega_{\mathrm{r}}^{2}} \cdot \frac{Z_{\mathrm{CS} 2}}{Z_{\mathrm{CS} 2}+Z_{\mathrm{m} 2}}
$$

Here $Z_{\mathrm{cs} 2}$ and $Z_{\mathrm{m} 2}$ are the impedance of damping capacitance, $C_{\mathrm{s}}$, and the second motional impedance at $2 \omega_{\mathrm{r}}$. Since $Z_{\mathrm{cs} 2}$ and $Z_{\mathrm{m} 2}$ can be expressed as Eqs. (7) and (8), Eq. (6) is rewritten as Eq. (9).

$$
\begin{aligned}
& Z_{\mathrm{CS} 2}=\frac{1}{j 2 \omega_{\mathrm{r}} C_{\mathrm{s}}} \\
& Z_{\mathrm{m} 2}=R_{2}+j\left(2 \omega_{\mathrm{r}} L_{2}-\frac{1}{2 \omega_{\mathrm{r}} C_{2}}\right) \\
& V_{\mathrm{h} 2 \mathrm{ap}}=\frac{\gamma_{\mathrm{D} 31}^{\prime} I_{3}^{2}}{2 \omega_{\mathrm{r}}^{2}} \cdot \frac{1}{1-2 \omega_{\mathrm{r}} C_{\mathrm{s}}\left(2 \omega_{\mathrm{r}} L_{2}-\frac{1}{2 \omega_{\mathrm{r}} C_{2}}\right)+j 2 \omega_{\mathrm{r}} C_{\mathrm{s}} R_{2}}
\end{aligned}
$$

Equation (9) shows that the $V_{\text {h2ap }}$ depends greatly on where the frequency of $2 \omega_{\mathrm{r}}$ is on the second harmonic resonance curve. When $2 \omega_{\mathrm{r}}$ equals $\omega_{2 \mathrm{a}}=2 \pi f_{2 \mathrm{a}}$, it is expressed as

$$
2 \omega_{\mathrm{r}} C_{\mathrm{s}}\left(2 \omega_{\mathrm{r}} L_{2}-\frac{1}{2 \omega_{\mathrm{r}} C_{2}}\right)=1
$$

$V_{\text {h2ap }}$ shows therefore the maximum value. The curve fitted to the results of Fig. 3(b), obtained by substituting the equivalent circuit constants of the second harmonic resonance of a sample used in the measurement of $V_{\text {h2ap }}$ in Fig. 3(b) $\left(C_{\mathrm{s}}=\right.$ $\left.759 \mathrm{pF}, R_{2}=33.1 \Omega, L_{2}=39.6 \mathrm{mH}, C_{2}=46.4 \mathrm{pF}\right)$ and an adequate value of $\gamma_{\mathrm{D} 31}^{\prime}\left(6.5 \times 10^{14} \mathrm{~V} / \mathrm{C}^{2}\right)$ into Eq. (9), is shown as a solid line in Fig. 3(b). The fitted curve agrees well with the measured values. The results are the same as the case of $\xi_{\mathrm{D} 31}$. Thus, by inserting the nonlinear voltage source, the change of $V_{\text {h2ap }}$ can be quantitatively explained.

The samples different in width, $w$, and equal in length and thickness $(l=29.5 \mathrm{~mm}, b=0.9 \mathrm{~mm})$ of Fig. $1(\mathrm{~b})$ were driven at each resonance angular frequency, $\omega_{\mathrm{r}}$, by the constant current method. The magnitudes of second-harmonic voltage appearing between electrodes, $V_{\mathrm{h} 2 \mathrm{ap}}$, were measured at various driving currents, $I_{3}$. Figure 5 shows $\gamma_{\mathrm{D} 31}^{\prime}$ values obtained by substituting $V_{\text {h2ap }}$ and $I_{3}$ values into Eq. (9). The horizontal axis is shown by average current densities $\left(2 I_{3} / w l\right)$. Since $\gamma_{\mathrm{D} 31}^{\prime}$ represents the quantitative relation between $V_{\mathrm{h} 2 a \mathrm{p}}$ and $I_{3}$ as shown in Eq. (6), $\gamma_{\mathrm{D} 31}^{\prime}$ shows different values if electrode surface differs. Increasing electrode surface, decreasing $\gamma_{\mathrm{D} 31}^{\prime}$ value. The purpose of the paper is to theoretically analyze the quantitative relationship between the effective nonlinear coefficient, $\gamma_{\mathrm{D} 31}^{\prime}$, and the nonlinear coefficient as a material constant, $\gamma_{\mathrm{D} 31}$.

\section{Theoretical analysis}

The current density and electric flux density in a rectangular bar are distributed with the cosine distribution in the length direction of the sample driven at the fundamental resonance angular frequency, $\omega_{\mathrm{r}}$, by the constant-current method. Expressing $x$-distance, where the origin is at the center of the sample, $D_{3}$ is expressed

$$
D_{3}=-D_{0} \cos \frac{\pi}{l} x \cdot \cos \omega_{\mathrm{r}} t
$$

where, $x$ is restricted in the region of electrode $(0 \leqq x \leqq l / 2)$. The nonlinear voltage generated in the sample due to the

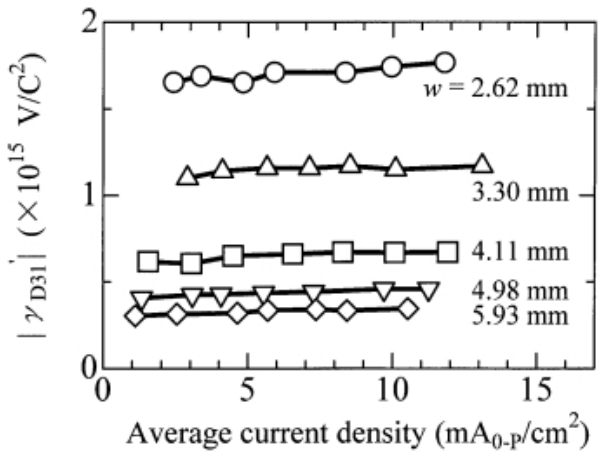

Fig. 5. Effective nonlinear coefficient of second-higher term $\gamma_{\mathrm{D} 31}^{\prime}$ as a function of average current density in the samples with different sample width.

second-higher term is expressed as

$$
v_{\gamma 31}=b \gamma_{\mathrm{D} 31} D_{3}^{2}=b \gamma_{\mathrm{D} 31} D_{0}^{2} \cos ^{2} \frac{\pi}{l} x \cdot \cos ^{2} \omega_{\mathrm{r}} t,
$$

where $b$ is the thickness of the sample. Expressing current density as $j_{3}=\partial D_{3} / \partial t$, the sample current, $i_{3}$, is expressed as

$$
i_{3}=w \int_{0}^{l / 2} j_{3} \mathrm{~d} x=\frac{l w \omega_{\mathrm{r}} D_{0}}{\pi} \sin \omega_{\mathrm{r}} t=I_{3} \sin \omega_{\mathrm{r}} t .
$$

From Eq. (13), we obtain

$$
D_{0}=\frac{I_{3} \pi}{l w \omega_{\mathrm{r}}} .
$$

Substituting Eq. (14) into Eq. (12), we get

$$
\begin{aligned}
v_{\gamma 31}= & b \gamma_{\mathrm{D} 31} \frac{I_{3}^{2} \pi^{2}}{l^{2} w^{2} \omega_{\mathrm{r}}^{2}} \cos ^{2} \frac{\pi}{l} x \cdot \cos ^{2} \omega_{\mathrm{r}} t \\
= & b \gamma_{\mathrm{D} 31} \frac{I_{3}^{2} \pi^{2}}{4 l^{2} w^{2} \omega_{\mathrm{r}}^{2}}\left\{1+\cos \frac{2 \pi}{l} x+\left(1+\cos \frac{2 \pi}{l} x\right)\right. \\
& \left.\times \cos 2 \omega_{\mathrm{r}} t\right\} .
\end{aligned}
$$

From Eq. (15), the magnitude of the second-harmonic voltage generating in the sample is expressed as

$$
V_{\mathrm{h} 2}(x)=b \gamma_{\mathrm{D} 31} \frac{I_{3}^{2} \pi^{2}}{4 l^{2} w^{2} \omega_{\mathrm{r}}^{2}}\left(1+\cos \frac{2 \pi}{l} x\right) .
$$

It is necessary to investigate how second-harmonic voltage is measured between electrodes when the second-harmonic voltage with cosine distribution generates in the sample having electrodes on the upper and lower surfaces. Since $2 \omega_{\mathrm{r}}$ is under the influence of second-harmonic piezoelectric resonance, the distribution of motional impedance consequently exists in the sample. The impedance against the second-harmonic voltage has reactance components since the second-harmonic resonance angular frequency, $\omega_{2 \mathrm{r}}$, is different from $2 \omega_{\mathrm{r}}$. The impedance per meter in the length direction, $Z_{\mathrm{m} 2}(x)$, is expressed as

$$
Z_{\mathrm{m} 2}(x)=Z_{\mathrm{m} 2}(l / 4) \frac{1}{\sin \frac{2 \pi}{l} x}
$$

where $Z_{\mathrm{m} 2}(l / 4)$ is the impedance per meter at $x=l / 4$.

In the sample having distributions of both second-harmonic electromotive force and impedance, the voltage measured between electrodes can be calculated using Millman's theorem. ${ }^{15}$ ) 


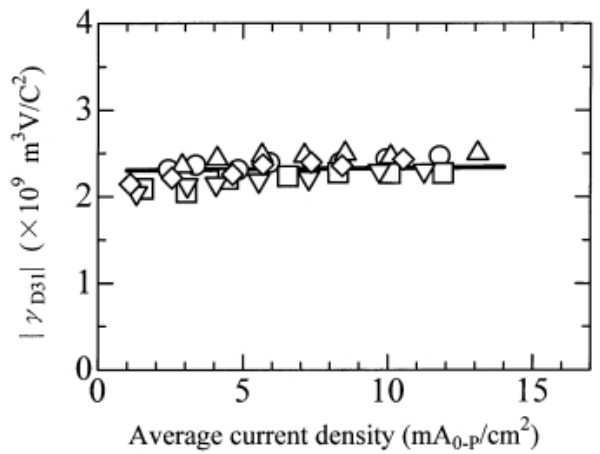

Fig. 6. Nonlinear coefficient of second-higher term $\gamma_{\mathrm{D} 31}$ as a function of average current density in the samples with different sample width.

On the other hand, in the case of $2 \omega_{\mathrm{r}} \neq \omega_{2 \mathrm{r}}$, the influence of $Z_{\mathrm{CS} 2}$ can be considered. Considering the influence of $Z_{\mathrm{CS} 2}$ and using Millman's theorem, the magnitude of the second-harmonic voltage appearing between electrodes is expressed as

$$
\begin{aligned}
V_{\mathrm{h} 2 \mathrm{ap}} & =\frac{\int_{0}^{l / 2} \frac{V_{\mathrm{h} 2}(x)}{Z_{\mathrm{m} 2}(x)} \mathrm{d} x}{\int_{0}^{l / 2} \frac{1}{Z_{\mathrm{m} 2}(x)} \mathrm{d} x} \cdot \frac{Z_{\mathrm{CS} 2}}{Z_{\mathrm{CS} 2}+Z_{\mathrm{m} 2}} \\
& =b \gamma_{\mathrm{D} 31} \frac{I_{3}^{2} \pi^{2}}{4 l^{2} w^{2} \omega_{\mathrm{r}}^{2}} \cdot \frac{Z_{\mathrm{CS} 2}}{Z_{\mathrm{CS} 2}+Z_{\mathrm{m} 2}} .
\end{aligned}
$$

Comparing Eq. (6) with Eq. (18), as the relation of $\gamma_{\mathrm{D} 31}$ and $\gamma_{\mathrm{D} 31}^{\prime}$, we get

$$
\gamma_{\mathrm{D} 31}=\frac{2 l^{2} w^{2}}{b \pi^{2}} \gamma_{\mathrm{D} 31}^{\prime}
$$

By converting $\gamma_{\mathrm{D} 31}^{\prime}$ values in Fig. 5 into $\gamma_{\mathrm{D} 31}$ values using Eq. (19), Fig. 6 is obtained. The $\gamma_{D 31}$ values converge with around a constant value $\left(\gamma_{\mathrm{D} 31}=2.3 \times 10^{9} \mathrm{~m}^{3} \mathrm{~V} / \mathrm{C}^{2}\right)$. This fact demonstrates that the analysis in the present paper is valid.

\section{Conclusions}

When a piezoelectric rectangular bar with half electrodes was driven at the fundamental resonance frequency by the constant-current method, nonlinear second-harmonic voltage appearing between electrodes due to $\gamma_{\mathrm{D} 31}$ are expressed as Eq. (18). The nonlinear coefficient of the second-higher term, $\gamma_{\mathrm{D} 31}$, can be therefore derived as a material constant by measuring the magnitude of the nonlinear second-harmonic voltage appearing between electrodes and using the value into Eq. (18).

\section{References}

1) Ishii, K., Akimoto, N., Tashiro, S. and Igarashi, H., J.Ceram. Soc. Japan, Vol. 106, pp. 555-558 (1998).

2) Ishii, K., Yamada, T., Tashiro, S. and Igarashi, H., Trans. Mat. Res. Soc. Jpn., Vol. 24, pp. 23-26 (1999).

3 ) Tashiro, S., Murata, T., Ishii, K. and Igarashi, H., Jpn. J. Appl., Phys., Vol. 40, pp. 5679-5682 (2001).

4) Tashiro, S., Ishii, K. and Nagata, K., Jpn. J. Appl. Phys., Vol. 42, pp. 6068-6073 (2003).

5) Cho, Y. and Matsuno, F., Trans. IEICE, Vol. 76-A, pp. 153-160 (1993) [in Japanese].

6) Beige, H. and Schmidt, G., Ferroelectrics, Vol. 41, pp. 39-49 (1982).

7) Kugel, V. D. and Cross, L. E., J. Appl. Phys., Vol. 84, pp. 2815-2830 (1998).

8) Damjanovic, D., J. Appl. Phys., Vol. 82, pp. 1788-1797 (1997).

9) Umeda, M., Nakamura, K. and Ueha, S., Jpn. J. Appl. Phys., Vol. 37, pp. 5322-5325 (1998).

10) Umeda, M., Nakamura, K. and Ueha, S., Jpn. J. Appl. Phys., Vol. 38, pp. 5581-5585 (1999).

11) Takahashi, S., Yamamoto, M. and Sasaki, Y., Jpn. J. Appl. Phys., Vol. 37, pp. 5292-5296 (1998).

12) Umeda, M., Nakamura, K., Takahashi, S. and Ueha, S., Jpn. J. Appl. Phys., Vol. 39, pp. 5623-5628 (2000).

13) Tashiro, S., Ishii, K. and Nagata, K., J. Ceram. Soc. Japan, Vol. 110, pp. 649-655 (2002)

14) Ishii, K., Tashiro, S. and Nagata, K., Trans. Mat. Res. Soc. Jpn., Vol. 27, pp. 265-268 (2002).

15) Toba, K., "Kisodenkikairo (Basic Electrical Circuit)," Koronasha, Tokyo (1985) pp. 27-28 [in Japanese]. 